\title{
Frontières
}

\section{Lire désespérément... W.G. Sebald}

\section{Frédérique Bernier}

Volume 21, numéro 2, printemps 2009

Détresse psychique et antidépresseurs

URI : https://id.erudit.org/iderudit/039458ar

DOI : https://doi.org/10.7202/039458ar

Aller au sommaire du numéro

Éditeur(s)

Université du Québec à Montréal

ISSN

1180-3479 (imprimé)

1916-0976 (numérique)

Découvrir la revue

Citer cet article

Bernier, F. (2009). Lire désespérément... W.G. Sebald. Frontières, 21(2), 51-55. https://doi.org/10.7202/039458ar

\section{Résumé de l'article}

Dans la foulée d’Évelyne Grossman et de sa réflexion sur « la paradoxale vitalité de la négativité dépressive " (L'angoisse de penser, 2008), cet article envisage l'exploration littéraire de la négativité et de la dépossession de soi, caractéristique d'une certaine modernité que l'on peut faire remonter à Mallarmé, en tant qu'elle peut fonctionner, pour le lecteur, à la manière d'un antidépresseur paradoxal. Questionnant d'abord de façon générale certaines conceptions « sublimantes ", réparatrices ou rédemptrices de la littérature (Leo Bersani) et la prégnance des modèles platoniciens et aristotéliciens de la création comme pharmakon, l'auteure tente ensuite de cerner plus spécifiquement, à partir de l'oeuvre de l'écrivain allemand W.G. Sebald, le caractère tout à la fois anxiogène et libérateur de la symbolisation de la perte en littérature.
Ce document est protégé par la loi sur le droit d'auteur. L'utilisation des services d'Érudit (y compris la reproduction) est assujettie à sa politique d'utilisation que vous pouvez consulter en ligne.

https://apropos.erudit.org/fr/usagers/politique-dutilisation/ 


\section{Résumé}

Dans la foulée d'Évelyne Grossman et de sa réflexion sur «la paradoxale vitalité de la négativité dépressive " (L'angoisse de penser, 2008), cet article envisage l'exploration littéraire de la négativité et de la dépossession de soi, caractéristique d'une certaine modernité que l'on peut faire remonter à Mallarmé, en tant qu'elle peut fonctionner, pour le lecteur, à la manière $d^{\prime} u n$ antidépresseur paradoxal. Questionnant d'abord de façon générale certaines conceptions "sublimantes", réparatrices ou rédemptrices de la littérature (Leo Bersani) et la prégnance des modèles platoniciens et aristotéliciens de la création comme pharmakon, I'auteure tente ensuite de cerner plus spécifiquement, à partir de l'œuvre de l'écrivain allemand W.G. Sebald, le caractère tout à la fois anxiogène et libérateur de la symbolisation de la perte en littérature.

Mots clés: littérature - négativité antidépresseur - W.G. Sebald.

\section{Abstract}

Following Évelyne Grossman and her developments about the "paradoxical vitality of depressive negativity" (L'angoisse de penser, 2008), this article addresses the literary exploration of negativity and self-deprivation, characteristic of a certain modernity one can retrace up to Mallarmé, and proposes that it can function, for the reader, as a paradoxical antidepressant. Questioning at first more generally the current sublimating conceptions of literature and the impregnation of the platonician and aristotelian models of creation as pharmakon, the author seeks then to embrace more precisely, on the basis of the works by German writer W.G. Sebald, the all-together anguishing and liberating effect of the symbolization of loss in literature.

Keywords: literature - negativity antidepressant-W.G. Sebald.

\section{LIRE DÉSESPÉRÉMENT... W.G. SEBALD}

Frédérique Bernier, Ph. D., stagiaire postdoctorale, Department of Romance Languages and Literatures, Université Harvard.

Des livres comme ceux de W.G. Sebald résistent de façon évidente à l'injonction de vitalité et à la fonction guérisseuse, réparatrice ou «rédemptrice» auxquelles on reconduit le plus souvent la culture ${ }^{1}$. Les ruines sur lesquelles ces livres s'édifient, à travers lesquelles ils nous font séjourner tels des fantômes, nous poussent à penser de façon tout autre le travail de l'art, en un lieu et selon une logique qui, loin de prétendre à l'apaisement, consiste au contraire à sonder à quel point il n'y a de création et de vie possible qu'à rappeler sans cesse le caractère fondateur de la négativité, de la perte et de la mort, dans l'histoire mondiale comme dans la vie psychique. Chez Sebald, ce sont les vertiges de la dépersonnalisation, les chaînes d'identification miroitantes entre les morts et les vivants, les échos déroutants entre les êtres imaginaires et les situations historiques, tous ces phénomènes confinant à l'hallucination dans lesquels Platon aurait vu le comble du mensonge et de l'illusion, qui font qu'une vérité littéraire semble pouvoir être retrouvée, là même où la littérature consent à perdre son identité et ses repères.
Partant de la conception mallarméenne de la lecture, fondatrice d'une certaine négativité littéraire moderne, et convoquant les mimèsis pharmaceutiques contradictoires de Platon et d'Aristote, on tentera ici d'examiner comment, lisant ces livres à première vue désespérants, le lecteur trouve peut-être à se soigner autrement - en deçà de toute fabulation compensatrice.

\section{DÉSESPÉRER AVEC MALLARMÉ}

«Strictement j'envisage, écartés vos folios d'études, rubriques, parchemin, la lecture comme une pratique désespérée", écrit Mallarmé dans La Musique et les Lettres (1976, p. 355). Poursuite d'une «autre chose» toujours absente, désespérément fuyante, la lecture, pour mélancolique ou déprimée qu'elle apparaisse déjà sous la plume de Mallarmé ("La chair est triste hélas, et j'ai lu tous les livres», dit un des poèmes les plus célèbres du poète [1977]), n'en est pas moins envisagée dans ces premières lignes comme une pratique active, comme un espace de jeu, de projections et d'appropriations (variante du Fort- $D a$ freudien) qui dégage le sujet de la "prépondérance» tautologique des choses («certes, n'est que ce qui est») pour l'engager dans la ronde sans fin de l'absence, 
C'EST PEUT-ÊTRE SEULEMENT À CONDITION DE POUVOIR SE DONNER

TOUT ENTIER, DE SE PERDRE ABSOLUMENT, QU'UN LIVRE OU

QU'UN TEXTE PEUT FONCTIONNER COMME UN ANTIDÉPRESSEUR

INATTENDU - OU COMME UN ESPACE THÉRAPEUTIQUE PARADOXAL.

en une «mentale poursuite» où la solitude s'avère tour à tour infiniment vide et peuplée, ennuyée et exaltée :

À quoi sert cela - À un jeu. En vue qu'une attirance supérieure comme d'un vide, nous avons droit, le tirant de nous par de l'ennui à l'égard des choses si elles s'établissaient solides et prépondérantes - éperdument les détache jusqu'à s'en remplir et aussi les douer de resplendissement, à travers l'espace vacant, en des fêtes à volonté et solitaires. (Mallarmé, 1976, p. 356.)

La lecture s'expose ici comme une affaire reposant tout entière sur l'entretien d'un vide; une pratique mélancolique, donc, mais pour autant non moins féconde, voire vitale, en ce que ce vide relance infiniment le désir, jusqu'à se confondre avec son mouvement, alors qu'il se rapporte à un objet - le livre, le texte, les lettres - qui ne lui est jamais tout à fait ajusté et qui pourtant se présente comme idéal. Lieu idéal, le livre l'est peut-être précisément parce qu'il est non-lieu, espace indéfini où s'ébauche une quête personnelle d'impersonnalisation toujours forcément inaboutie et, par là même, dynamique: «Ainsi toute industrie a-t-elle failli à la fabrication du Bonheur, que l'agencement ne s'en trouve à portée: je connais des instants où quoi que ce soit, au nom d'une disposition secrète, ne doit satisfaire » (Mallarmé, 1976, p. 355).

Ce que les mots de Mallarmé illustrent, c'est peut-être à quel point l'activité littéraire moderne est hantée par une négativité (celle, notamment, de la mort du sujet, dont le poète a été un des premiers penseurs) qui, aussi mortifère et dépressive qu'elle puisse sembler de prime abord, s'avère potentiellement libératrice. Si, comme le pense Évelyne Grossman, une bonne partie de la littérature et de la philosophie du XX $\mathrm{X}^{\mathrm{e}}$ siècle est, à la suite de Mallarmé, traversée par «une négativité pure en quelque sorte, toujours ouverte, sans totalité ni synthèse, inépuisable, infiniment relancée, à la fois fidèle et infidèle à la pensée hégélienne» (Grossman, 2008, p. 23), il faut pouvoir envisager, avec Grossman toujours, «la paradoxale vitalité de la négativité dépressive» (2008, p. 25). Ce que Nancy Huston a, me semble-t-il, étrangement failli à penser dans Professeurs de désespoir (2004), où elle intente le procès des fauteurs de négativité, c'est comment le désespoir luimême peut être porteur de potentialités vitales dès lors qu'on lui offre un espace où se déployer, où se déporter infiniment... jusqu'à ce qu'il puisse ne plus se reconnaître, n'être plus lui-même. Si l'écriture est ainsi envisagée par Grossman et par les écrivains sur lesquels elle se penche (Blanchot, Bataille, Klossowski, Artaud, Beckett) comme un lieu où la négativité engage des "défigurations» (Grossman, 2004), des désintrications identitaires salutaires, il me semble que la lecture littéraire - c'est-à-dire une lecture qui ne cherche pas l'information, mais peut-être la «déformation »... - doit pouvoir être pensée non seulement comme le corollaire de cette négativité créatrice, mais peut-être comme son lieu premier. La lecture m'apparaît effectivement comme l'activité (la passivité ?) par excellence où s'opèrent des transferts d'identité et d'affects, des dépersonnalisations, des identifications projectives, des spectralisations, toutes sortes de jeux de doubles et d'échanges qui font du lecteur un être étrangement habité par des mondes, par des mouvements psychiques qui, tout à la fois, lui appartiennent et ne lui appartiennent pas, sont et ne sont pas de lui - un être hanté, intoxiqué.

Cela, pour peu que l'on s'engage véritablement - c'est-à-dire désespérément, comme Mallarmé, avec l'énergie du désespoir - dans l'acte de lire, pour peu que l'on y poursuive ce quelque chose ou ce quelqu'un d'autre qui n'y est jamais vraiment. Activité de dépressif, alors, la lecture? Peut-être, si la dépression consiste à ressentir très fort la "fatigue d'être soi ", si elle suppose le désir de se perdre, de se débarrasser, enfin, de soi, au moins momentanément; le désir d'être porté par le désir de l'autre comme s'il s'agissait du nôtre, quitte à passer d'abord par la dissociation, à payer le prix de l'angoisse. C'est peut-être seulement à condition de pouvoir se donner tout entier, de se perdre absolument, qu'un livre ou qu'un texte peut fonctionner comme un antidépresseur inattendu - ou comme un espace thérapeutique paradoxal. Paradoxal et rare, capricieux. Non-lieu, le livre, le texte, les lettres le sont aussi parce qu'ils ne cessent, le plus souvent, de résister à être un lieu pour soi, parce qu'ils sont la plupart du temps ennuyeux ou opaques, ou inutilement bavards, ou terriblement étrangers, n'offrant aucune prise à la déprise de soi. Désespérément refermés sur eux-mêmes, les livres attendent peut-être aussi qu'on les délivre en projetant sur eux notre propre perte. Encore faut-il qu'on y adhère suffisamment pour s'y laisser choir. Peutêtre trouve-t-on le bon livre comme on trouve le bon objet, la bonne molécule, le bon cocktail de neurotransmetteurs, ce qui est à même de nous déprendre, d'une manière ou d'une autre, de l'étroitesse parfois désespérante de nos schèmes mentaux. Étrange antidépresseur pourtant, qui brouille l'identité plutôt que d'en restaurer l'unité.

\section{S'INTOXIQUER AVEC PLATON ET ARISTOTE}

On sait que l'écriture et la lecture ont été pensées très tôt dans les termes de la pharmacopée, et ce pour relever précisément le caractère peu sûr, instable, du remède scripturaire, toujours susceptible de se renverser en son contraire, de passer - l'ambiguité est constitutive du mot grec pharmakon - de remède à poison ${ }^{2}$. Dans le Phèdre de Platon, toute écriture, aussi utile qu'elle paraisse de prime abord pour préserver le savoir et pallier les défauts de la mémoire, s'avère porteuse d'un danger qui tient à la coupure qu'elle instaure entre elle et l'auteur, et qui fait d'elle quelque chose d'à la fois étrangement bavard et muet, fixe et mobile, quelque chose de l'ordre $\mathrm{du}$ mort-vivant, d'une «semblance» de vie proprement unheimlich $^{3}$. Mais ce qui inquiète Platon dans l'écriture tient peutêtre surtout à son caractère vagabond, à son adresse incertaine ${ }^{4}$. L'écriture, dans la mesure où elle échappe par définition à son auteur et où, orpheline, elle peut faire l'objet de toutes les appropriations, de toutes les interprétations, même les plus folles, est d'emblée suspecte. C'est, en fait, la possibilité d'une mauvaise lecture qui la gangrène, qui la contamine dès le départ. Les déformations, les détournements sont en germe dans l'écriture, pour Platon, comme ils le sont dans la mimèsis, car toutes deux sont du côté du double spectral, du mensonge, de la fausse vitalité. Toute l'histoire de la littérature (et celle de la philosophie) se résume peut-être à l'alternance entre deux conceptions de la représentation et de la création, entre la pensée platonicienne, qui fait de celle-ci une production dégradée, ouvrant le royaume de l'illusion, des dédoublements trompeurs, et la pensée aristotélicienne, qui en fait la production par excellence, prolongeant la création divine, remédiant aux défauts de la Nature et purgeant l'âme de ses passions ${ }^{5}$. S'agissant tantôt du créateur tantôt du lecteur, l'espace littéraire ne cesse de superposer les métaphores 
pharmaceutiques ou thérapeutiques et religieuses, balançant indéfiniment entre mal et guérison, entre faute et réparation. Les modèles platoniciens et aristotéliciens continuent ainsi à être singulièrement actifs dans la littérature moderne et contemporaine, et ce, sous une forme étrangement intriquée, comme c'est le cas à travers toute l'œuvre en prose de Beckett, où l'espace du langage et de la fiction se présente tout à la fois comme le lieu de la perdition (en même temps perte de soi et faute) et celui où l'on ne cesse néanmoins de chercher un salut, une rédemption. On retrouve sous la plume d'un auteur comme Michel Leiris une même ambivalence, une même méfiance, exprimée cette fois dans les termes de la pharmacopée qui étaient ceux de Platon: "L'une de nos grandes faiblesses ne consiste-t-elle pas à compter sur l'écriture comme un médicament alors qu'elle n'est qu'une drogue?» (cité par Harel, 1994, p. 48).

Jusque dans la structure même d'une réflexion psychanalytique comme celle de Simon Harel dans L'écriture réparatrice (1994), inspirée des travaux de Melanie Klein, on constate à quel point il est difficile de se défaire de ce balancement, peutêtre constitutif du psychisme lui-même, entre un fantasme de restauration et une pulsion mortifère qui ne cessent d'être tour à tour attribués aux sources de la création littéraire. Leo Bersani (1986) a bien montré à quel point la notion de sublimation (chez Freud et chez Klein) est elle-même traversée par cette ambivalence que la théorie psychanalytique tend à vouloir surmonter en soutenant une conception foncièrement rédemptrice de la culture, l'idée d'un art qui puisse en fin de compte, telle la catharsis aristotélicienne, réparer les ravages du désir et de la pulsion de mort. Or, assigner à l'art une telle fonction restauratrice revient, comme le souligne Bersani (1986, p. 414), à lui donner une mission morale et normative et surtout à dénier le travail esthétique de la négativité en privilégiant un modèle artistique empreint de positivité et de plénitude (l'œuvre faisant office de bonne mère contenante) que la modernité a pour une bonne part mis a mal ${ }^{6}$.

Des livres comme ceux de W.G. Sebald m'apparaissent résister effectivement de toute leur force à cette injonction d'unité, de sublimation et de restauration. Si une forme de sublimation demeure à l'œuvre chez Sebald, c'en est une qui, loin de colmater la brèche, tend plutôt à explorer - et l'on retrouve ici le lecteur mallarméen - les vides et les absences; à vérifier sans cesse la précarité du réel et des identités. La littérature est le lieu de cette vérification qui prend parfois les allures d'une intoxication ${ }^{7}$. Platon et Aristote nous accom- pagnent toujours à travers ces œuvres où puissance et impuissance, prodiges et maléfices, guérison et rechute, se renversent sans cesse l'un dans l'autre.

\section{SE PERDRE AVEC SEBALD}

Lire les livres de Sebald, tels Vertiges (2001a), Les émigrants (2001b) ou Les anneaux de Saturne $(1999)^{8}$, c'est suivre le mouvement d'une prose dont la singularité est sans doute d'être à la fois ferme et poreuse, transparente et vertigineuse. Le plus souvent objective et anonyme, la voix de Sebald collige des faits divers et des bouts de fiction, rapporte des épisodes historiques et des découvertes savantes, opère d'étonnants rapprochements entre des destins singuliers, marqués par la perte, la folie et la disparition. Cette voix apparaît d'autres fois dans des passages et des chapitres où le Je survient inopinément, tout à coup profondément subjective et incarnée, s'avérant elle-même entraînée dans le vertige des identifications et des coïncidences, révélant à quel point l'érudition et l'accumulation des références littéraires et historiques, loin d'être ici une activité issue de la maîtrise, qu'elle soit savante ou ludique, engage l'identité d'un sujet qui semble tantôt s'y perdre tantôt y reprendre pied momentanément. Le va-et-vient entre les époques, entre l'histoire et la fiction, entre les rêveries et la notation scrupuleuse des faits et des dates, se superpose à l'errance géographique du narrateur, qui se promène de lieu en lieu, à pied ou en train, comme dans Vertiges, livre dans lequel le narrateur voyage entre l'Angleterre (où Sebald s'est exilé très tôt pour gagner sa vie comme professeur de littérature à l'Université de Norwich, jusqu'à sa mort accidentelle en 2001), l'Allemagne (lieu d'origine de Sebald, né en 1944 dans un village de Bavière, ce terreau du nazisme dont l'ombre plane sur tous ses livres), l'Autriche et l'Italie, où l'accompagnent les spectres de Dante, de Stendhal, de Kafka et de Casanova. Ici le voyage, qui donne sa trame vagabonde à l'écriture, prend lui-même la forme d'un pharmakon, faisant basculer l'espoir de rétablissement en constat d'aggravation.

En octobre 1980, partant d'Angle-

terre, où je vis depuis près de vingtcinq ans dans un comté la plupart du temps enfoui sous les nuages gris, j'étais allé à Vienne, dans l'espoir qu'un changement de lieu me permettrait de surmonter une passe particulièrement difficile. Mais à Vienne il s'avéra dès mon arrivée que les journées, dépourvues à présent des tâches habituelles d'écriture et de jardinage, m'apparurent interminables et que je ne savais plus à quoi me vouer (Sebald, 2001a, p. 41).
Le narrateur, errant dans les rues du matin au soir, se laisse à ce point imprégner par les lieux et les êtres qui l'entourent qu'il finit par sombrer, en proie à des vertiges, des hallucinations («Un jour, dans la Gonzagagasse, je crus même reconnaître le poète Dante, menacé du bûcher et banni de sa ville» [Sebald, 2001a, p. 43]) et à une clochardisation progressive qui le rapproche étonnamment, non seulement des auteurs qui le préoccupent et dont il reprend consciemment les itinéraires, mais aussi d'un autre personnage littéraire à l'identité poreuse et vacillante, le Malte Laurids Brigge des Carnets de Rainer Maria Rilke (1995), avec lequel le narrateur sebaldien partage le sentiment d'une appartenance de plus en plus visible à la communauté des réprouvés :

Mes longs séjours sur les bancs publics, mes errances à travers la ville, ma propension de plus en plus marquée à éviter aussi les restaurants et à prendre mes repas debout à un kiosque ou en plongeant directement dans le pochon de papier, au gré des circonstances, tout cela avait commencé sans que je m'en rende compte à faire de moi quelqu'un d'autre. La poussière sur mes vêtements et mon aspect négligé entraient de plus en plus en contradiction avec le fait que je continuais à loger dans un hôtel. Je me mis à trimbaler avec moi un sac plastique rapporté d'Angleterre où je fourrais toutes sortes de choses inutiles, des choses dont sans me l'avouer je finissais par ne plus pouvoir me séparer. Revenant à une heure tardive de mes excursions, je sentais, tandis que j'attendais l'ascenseur dans le hall de l'hôtel en serrant dans mes bras en croix le sac contre ma poitrine, posé sur mon dos le regard interrogateur du portier de nuit (Sebald, 2001a, p. 44-45).

Ce narrateur qui progressivement s'altère, s' "étrange»-All'estero ("À l'étranger») est le titre de la section de Vertiges dont sont tirés ces passages - et que l'on sent pourtant si proche, dont l'angoisse est si palpable, est-il bien le même que celui de la section précédente qui décrivait à distance, de façon tout à fait neutre, sans du tout y prendre part ni marquer sa présence d'aucune manière, les pérégrinations et les amours de Stendhal? Le lecteur lui-même ne s'y reconnaît plus très bien. D'une structure complexe, brouillant les frontières entre les lieux, les époques, les genres littéraires et les voix narratives (tout à la fois essais et fictions, encyclopédies et autobiographies, carnets de voyages et méditation onirique), les livres de Sebald nous font plonger dans un espace où toutes 
les identités vacillent ${ }^{9}$, et où elles restent pourtant lestées du poids de l'histoire; où, également, la ligne ferme de l'écriture, la sobriété de style, là où il est question des phénomènes psychiques les plus déroutants, permet au lecteur de s'y retrouver tel qu'en lui-même il ne s'appartient plus.

On pourrait dire de cette œuvre qu'elle est étrangement impersonnelle, et ce en plusieurs sens à la fois : parce qu'elle est marquée par un encyclopédisme, par un souci du détail et de la précision et par un sens aigu de l'histoire collective; parce que son narrateur est lui-même en proie à une menace constante de dépersonnalisation; et parce que son écriture apparaît comme désaffectée, sans pathos aucun, strictement descriptive. C'est par son impersonnalité même que cette voix peut être reçue comme appartenant à tous, comme la mienne. Réunissant de façon

C'EST LA POSSIBILITÉ DE

\section{L'EFFACEMENT DU SENTIMENT}

D'APPARTENANCE À L'HUMAIN

QUI CONSTITUE PEUT-ÊTRE

\section{LE CFUR DE L'EXPÉRIENCE}

\section{HUMAINE.}

étonnante ces trois modes de l'impersonnalité (l'histoire, la psychose, le neutre), les livres de Sebald m'apparaissent comme de formidables explorations de ce que Pierre Fédida (2007) nomme le «déshumain», soit l'effondrement subit d'une expérience de l'humanité, fondée sur la ressemblance et la reconnaissance, "le sentiment d'une déchéance, quand sont en train de se défaire le visage, les mots, la voix, la reconnaissance même des réactions chez l'autre» (Fédida, 2007, p. 14). C'est la possibilité de l'effacement du sentiment d'appartenance à l'humain qui constitue peut-être le cœur de l'expérience humaine. D'une façon tout autre que Beckett, Artaud ou Blanchot, grands explorateurs de la négativité sur lesquels se penchent Évelyne Grossman et Pierre Fédida, Sebald nous plonge dans cette désaffection de l'humain qui recouvre chez lui une portée tout à la fois psychique, esthétique et historique (les camps, la guerre ne sont jamais loin). Chez lui, la déchéance et la disparition deviennent les marqueurs mêmes de la vie et de l'histoire humaine: "Au fil des années, j'en suis arrivé à la conclusion que la vie désormais naît de tout ce fracas, celle qui vient après nous et qui lentement nous mènera à notre perte, comme nous menons lentement à sa perte tout ce qui a été là longtemps avant nous » (Sebald, 2001a, p. 72). Le narrateur va lui-même jusqu'au bout de ce mouvement, il va à sa perte jusqu'à risquer la dépersonnalisation plutôt que de tenter de s'en prémunir, et c'est peut-être ce qui permet au lecteur de s'y retrouver - d'appartenir lui-même à la perte, d'en faire l'expérience à même le mouvement infiniment dépersonnalisant de la lecture. C'est cette expérience mélancolique du fracas, de la disparition, de l'«étrangement» du plus intime qui se trouve à la source de la communauté négative qui lie Sebald aux auteurs, aux êtres, aux personnages ainsi qu'aux paysages qui le traversent et le hantent, le dérobent à lui-même plus qu'ils ne l'accompagnent - communauté qui comprend aussi le lecteur, pour peu qu'il se laisse lui aussi mener à sa perte. Loin d'être ce dont on doit se remettre à tout prix, la perte (la nôtre et celle des autres en nous) serait-elle l'expérience qu'il faudrait pouvoir vivre enfin et sans fin? La littérature - une certaine littérature - pourrait-elle être le lieu privilégié de cette perte infinie?

Contrairement à ce que laisse entendre la quatrième de couverture de l'édition Actes Sud/Babel des Émigrants, qui regroupe les récits d'êtres exilés après la Shoah, il n'y a pas, me semble-t-il, de "temps retrouvé » chez Sebald. Pas de rédemption proustienne par la mémoire ou la littérature ${ }^{10}$, plutôt une mémoire dont les trous ne sont jamais comblés, qui laisse le sujet errer dans l'espace et dans le temps, sans attache véritable et sans jamais qu'il soit possible de se ressaisir de la perte. Sur les lieux de son enfance qu'il revisite après trente ans, le narrateur réalise ainsi à quel point le passé reste informe, incompréhensible, étranger et inappropriable:

Au fil des années je m'étais fait beaucoup d'idées, mais [...] au lieu de se décanter, les choses en étaient devenues encore plus énigmatiques qu'avant. Plus je rassemblais les images d'autrefois [...] et plus il devenait invraisemblable que le passé se soit présenté sous cette forme, car rien ne pouvait y être qualifié de normal; au contraire, la majeure partie de ce qui était arrivé était ridicule, et quand ce n'était pas ridicule, c'était à frémir d'effroi (Sebald, 2001a, p. 216).

Marqué par les villes en ruines qui constituèrent le décor très peu normal de cette enfance et frappé par la quasiabsence de ces ruines dans la littérature allemande de l'après-guerre (Sebald, 2005a), Sebald a fait des décombres et des lieux désaffectés l'espace même de son écriture, ruinant ainsi toute entreprise de sublimation littéraire, laquelle repose bien entendu non seulement sur un fantasme culturel de rédemption, mais aussi sur des formes particulières privilégiant la continuité et la résolution. Un certain usage de l'imaginaire et du récit comporte dans sa structure même une vision téléologique de l'histoire dont la ténacité symbolique ne témoigne peut-être, comme le suggère Bersani, que de la haine de la vie et de ce qu'elle comporte de discontinu et de douloureux. Loin d'offrir un gain compensatoire à la discontinuité et à la perte, les livres de Sebald prolongent plutôt, par le type d'éclatement qui les caractérise, le processus de la disparition et de l'étiolement. Là même où, du fait de leur hétérogénéité générique, ils paraissent être du côté de la recollection, du recollement des morceaux, mettant en présence des choses disparates "à la manière d'une nature morte", selon l'expression de Sebald lui-même (2005b, p. 200) ${ }^{11}$, favorisant les rencontres entre les êtres, les lieux et les événements, ces livres et leur tissu de connexions ne réparent rien. Les connexions sebaldiennes, à la différence peut-être des correspondances symbolistes, n'éclairent pour ainsi dire rien, sinon le caractère improbable et insondable de leur rapport, leur aspect profondément énigmatique et incompréhensible.

Si ces livres restituent ces connexions sans pour autant rien retrouver, peut-être est-ce parce qu'ils se situent sur le plan d'une expérience, celle du "déshumain» dont parle Fédida, où la restauration du sujet et de ses objets n'a pas de sens parce que ceux-ci auraient dès l'origine basculé dans l'irreconnaissable: "[d]u côté de la perte et du deuil, on a encore des objets, on a encore la possibilité de concevoir un objet. Du côté de la disparition, on est dans l'inconnu du devenir de soi et de l'objet» (Fédida, 2007, p. 14-15). S'ils baignent dans l'insensé, le sens dont sont néanmoins porteurs les livres de Sebald réside peut-être en ceci qu'ils tentent d'épouser eux-mêmes ce devenir inconnu (cette avancée dans l'inconnaissable) de ce qui disparaît.

Un tel mouvement ne peut être que paradoxal. Il bouleverse sans pour autant l'annuler complètement la fonction mémorielle de la littérature et du même coup son fantasme de réparation. L'écriture apparaît dès lors non pas comme une entreprise de conservation et de restauration, mais plutôt comme une façon de mener à sa perte, d'accompagner la disparition, de recueillir les traces de l'éloignement. Il s'agit pour ainsi dire de contenir la perte, sachant qu'on n'y remédie pas, pas plus que l'on ne remédie à une histoire humaine fondée sur la destruction, la combustion:

Notre propagation sur terre passe par la carbonisation des espèces végétales 
supérieures et, d'une manière plus générale, par l'incessante combustion de toutes substances combustibles. De la première lampe-tempête jusqu'aux réverbères du XVIII ${ }^{\mathrm{e}}$ siècle, et de la lueur des réverbères jusqu'au blême éclat des lampadaires qui éclairent les autoroutes belges, tout est combustion, et la combustion est le principe intime de tout objet fabriqué par nous. La confection d'un hameçon, la fabrication d'une tasse de porcelaine et la production d'une émission de télévision reposent au bout du compte sur le même processus de combustion. Les machines conçues par nous ont, comme nos corps et comme notre nostalgie, un cœur qui se consume lentement. Toute la civilisation humaine n'a jamais été rien d'autre qu'un phénomène d'ignition plus intense d'une heure à l'autre et dont personne ne sait jusqu'où il peut croître ni à partir de quand il commencera à décliner (Sebald, 1999, p. 220-221).

Obéissant eux-mêmes à ce principe intime de la combustion qui conjugue de façon insensée la disparition élocutoire mallarméenne et les fours crématoires, les livres de Sebald brillent de l'éclat igné de ce qui se consume. S'il y a quelque chose de vital et d'infiniment beau dans ces livres, c'est ce séjour au bord de l'anéantissement, c'est aussi l'effet de contenance paradoxale de cette écriture, qui se présente malgré tout, à rebours de toute fiction consolatrice, comme un dernier rempart contre cette désintégration totale qu'elle serre au plus près - là où la perte nous tient comme notre danger et notre bien le plus propre.

\section{Bibliographie}

BERSANI, L. (1986). " "The culture of redemption": Marcel Proust and Melanie Klein », Critical Inquiry, vol. XII, $\mathrm{n}^{\circ} 2$, p. 399-421.

DERRIDA, J. (1992). La pharmacie de Platon, dans PLATON, Phèdre, Paris, GFFlammarion, p. 257-387.

FÉDIDA, P. (2007). «Humain/Déshumain. L'oubli, l'effacement des traces, l'éradication subjective, la disparition », dans P. FÉDIDA et al. (dir.), Humain/Déshumain, Paris, Presses universitaires de France, coll. «Petite bibliothèque de psychanalyse», p. 11-124.

GROSSMAN, É. (2008). L'angoisse de penser, Paris, Minuit, coll. «Paradoxe».

GROSSMAN, É. (2004). La défiguration, Artaud-Beckett-Michaux, Paris, Minuit, coll. «Paradoxe».

HAREL, S. (1994). L'écriture réparatrice, Le défaut autobiographique (Leiris, Crevel, Artaud), Montréal, XYZ, coll. «Théorie et littérature».
HUSTON, N. (2004). Professeurs de désespoir, Arles/Montréal, Actes Sud/Leméac.

LACOUE-LABARTHE, P. (1986). L'imitation des modernes (Typographies 2), Paris, Galilée.

LEIRIS, M. (1992). Journal 1922-1989, Paris, Gallimard, coll. «NRF».

MALLARMÉ, S. (1977). "Brise marine», dans Poésies, Paris, Librairie générale française, coll. «Le livre de poche», p. 24.

MALLARMÉ, S. (1976). «La Musique et les Lettres ", dans Igitur, Divagations, Un coup de dés, Paris, Gallimard, coll. "Poésie», p. 349-371.

NEPVEU, P. (1998). Intérieurs du Nouveau Monde, Montréal, Boréal, coll. "Papiers collés ».

PLATON (1992). Phèdre, Paris, GFFlammarion.

RILKE, R.M. (1995). Les carnets de Malte Laurids Brigge, traduit de l'allemand par Claude Porcell, Paris, GF-Flammarion.

SEBALD, W.G. (2005A). «Between history and natural history. On the literary description of total destruction ", dans Campo Santo, trad. par Anthea Bell, New York, The Modern Library, p. 65-95.

SEBALD, W.G. (2005B). «An attempt at restitution", dans Campo Santo, trad. par Anthea Bell, New York, The Modern Library, p. 195-206.

SEBALD, W.G. (2001A [1990]). Vertiges, trad. par P. Charbonneau, Paris, Gallimard, coll. «Folio».

SEBALD, W.G. (2001B [1992]). Les émigrants, trad. par P. Charbonneau, Paris, Gallimard, coll. "Folio».

SEBALD, W.G. (1999 [1995]). Les anneaux de Saturne, trad. par B. Kreiss, Paris, Gallimard, coll. «Folio».

SLOTERDIJK, P. (2003). "Pour une philosophie de la sur-réaction ", dans Ni le soleil ni la mort. Jeu de piste sous la forme de dialogues avec Hans-Jürgen Heinrichs, Paris, Fayard, coll. «Pluriel», p. 7-51.

\section{Notes}

1. Voir Bersani, 1986 (j’y reviendrai).

2. Je renvoie ici à la fameuse analyse de Jacques Derrida sur le Phèdre de Platon (Derrida, 1992).

3. "Car à mon avis, ce qu'il y a de terrible, Phèdre [dit Socrate], c'est la ressemblance qu'entretient l'écriture avec la peinture. De fait, les êtres qu'engendre la peinture se tiennent debout comme s'ils étaient vivants ; mais qu'on les interroge, ils restent figés dans une pose solennelle et gardent le silence. Et il en va de même pour les discours » (Platon, 1992, p. 180).

4. " Autre chose: quand, une fois pour toutes, il a été écrit, chaque discours va rouler de droite et de gauche et passe indifféremment auprès de ceux qui s'y connaissent, comme auprès de ceux dont ce n'est point l'affaire; de plus, il ne sait pas quels sont ceux à qui il doit ou non s'adresser. Que par ailleurs s'élèvent à son sujet des voix discordantes et qu'il soit injustement injurié, il a toujours besoin du secours de son père; car il n'est capable ni de se défendre ni de se tirer d'affaire tout seul » (Platon, 1992, p. 180).

5. Sur la prégnance et la dialectique des schèmes aristotéliciens et platoniciens, je renvoie à Philippe Lacoue-Labarthe (1986).

6. «A fundamentally meaningless culture thus ennobles gravely damaged experience. Or, to put it in other terms, art must preserve what might be called a moral monumentality - a requirement which explains, I believe, much of the mistrust in the modern period of precisely those modern works which have more or less violently rejected any such edifying and petrifying functions. Claims for the high morality of art may conceal a deep horror of life» (Bersani, 1986, p. 414).

7. En ce sens, Sebald rejoint peut-être le philosophe Sloterdijk qui défend l'«intoxication volontaire », cette idée, inspirée de Nietzsche et de la médecine homéopathique, que l'écrivain doit s'adonner à l'écriture comme à une dangereuse "expérience psychosomatique », prenant sur lui «les infections de l'existence contemporaine et de l'histoire» (Sloterdijk, 2003, p. 10-11). Pourtant, Sloterdijk, qui considère que l'Allemagne s'est enlisée dans la mauvaise conscience, est on ne peut plus aux antipodes de Sebald quant à son rapport à l'histoire et à la mémoire.

8. Tous trois traduits de l'allemand (Schwindel. Gefühle, 1990; Die Ausgewandertern, 1992; Die Ringe des Saturn, 1995) et parus chez Actes Sud, puis dans la collection "Folio » chez Gallimard. Je ne parlerai pas ici d'Austerlitz (paru en 2002) qui, pour reprendre une bonne part des obsessions des livres précédents, le fait sous une forme franchement romanesque, rompant d'une certaine manière avec l'étrangeté générique qui est le propre des autres œuvres de Sebald et qui m'intéresse particulièrement.

9. Cet attachement au sac et aux objets d'un être de plus en plus désocialisé, dont le moi fuit, a des échos beckettiens. L'errance du narrateur me fait également songer à l'essai liminaire d'Intérieurs du Nouveau Monde de Pierre Nepveu (1998), où l'auteur erre dans les rues de San Francisco, croisant des êtres inquiétants, lui-même désœuvré et au bord de la dépersonnalisation: "Je sentais venir le temps où je me mettrais moi aussi, peut-être, à inventer et à balbutier des mots étranges, les yeux fixés sur un texte devenu illisible » (Nepveu, 1998, p. 18).

10. Quoique chez Proust lui-même, comme le montre admirablement Bersani, cette rédemption demeure extrêmement ambiguë: "In Proust, art simultaneously erases, repeats, and redeems life. Literary repetition is an annihilating salvation " (Bersani, 1986, p. 403).

11. Sebald décrit ainsi sa manière de faire (method of procedure): «engraving and linking together apparently disparate things in the manner of a still life» (Sebald, 2005b, p. 200). 